\title{
TITLE:
}

\section{Three-body hypernetted-chain equation and its numerical solution}

$\operatorname{AUTHOR}(S)$ :

Kim, K; Munakata, T

CITATION:

Kim, K ... [et al]. Three-body hypernetted-chain equation and its

numerical solution. JOURNAL OF CHEMICAL PHYSICS 2002, 117(1): 277 281

ISSUE DATE:

2002-07-01

URL:

http://hdl.handle.net/2433/50288

\section{RIGHT:}

Copyright 2002 American Institute of Physics. This article may be downloaded for personal use only. Any other use requires prior permission of the author and the American Institute of Physics. 


\title{
Three-body hypernetted-chain equation and its numerical solution
}

\author{
Kang Kim ${ }^{\text {a) }}$ and Toyonori Munakata ${ }^{\text {b) }}$ \\ Department of Applied Mathematics and Physics, Graduate School of Informatics, Kyoto University, \\ Kyoto 606-8501, Japan
}

(Received 11 February 2002; accepted 11 April 2002)

\begin{abstract}
The HNC (hypernetted-chain) equation is generalized at a three-body level based on the three-body density functional theory and the Percus' idea. Supplementing with the generalized OrnsteinZernike relations, we derive the three-body HNC theory for two- and three-body correlation functions. We solved the three-body HNC theory numerically for the case of a one-dimensional fluid and obtained the solution self-consistently. Our results are compared with the (two-body) HNC theory and molecular dynamics simulations. It is found that the three-body HNC theory improves the HNC one from the viewpoint of the radial distribution function. (C) 2002 American Institute of Physics. [DOI: 10.1063/1.1482704]
\end{abstract}

\section{INTRODUCTION}

The pair distribution function, which is usually called the radial distribution function $g_{2}(r)$, plays a central role in characterizing structures of simple liquids. ${ }^{1}$ The $g_{2}(r)$ has been obtained by neutron scattering experiments ${ }^{1}$ or computer simulations. ${ }^{2}$ Theoretically, there have been developed some equations for $g_{2}(r)$, such as the hypernetted-chain (HNC) equation or Percus-Yevick (PY) equation to mention a few, ${ }^{1,3}$ based on the density functional theory (DFT) and diagram methods. ${ }^{1}$ It is noted that these equations are usually of the form of integral equations and rather heavy numerical calculations are required to solve them, except for the PY equation for a hard-core system ${ }^{1}$ which allows an analytic solution.

As an exact theoretical framework and also as a practical and convenient tool to study the structure of liquids, the DFT has been playing an important role $^{4}$ in studies of the freezing, ${ }^{5,6}$ glass transition, ${ }^{7}$ molecular liquids, ${ }^{8}$ and so on. It is worth mentioning that the HNC equation for $g_{2}(r)$ can be derived from the DFT.

Let us consider a $d$-dimensional fluid system with an interaction potential $\phi(r)$. If one particle is located at the origin $\mathbf{r}=0$ of the system, the one-particle density can be related, with the aid of the Percus' idea, to the paircorrelation function. ${ }^{9}$ That is, the equilibrium density $n(\mathbf{r})$ represents $n_{0} g_{2}(r)$ with the uniform density $n_{0}$ and the normalization condition $g_{2}(r) \rightarrow 1$ as $r \rightarrow \infty$. According to the variational principle of the DFT, we obtain an equation to determine the equilibrium density $n(\mathbf{r})$,

$$
\frac{\delta F[n]}{\delta n(\mathbf{r})}+\phi(r)=\mu,
$$

where $F[n]$ is the free energy functional of a fluid and $\mu$ represents a chemical potential.

The free energy functional $F[n]$ is divided into the ideal gas part $F_{\text {id }}$ and the interaction part $F_{\text {ex }} \cdot F_{\text {id }}$ is given exactly

\footnotetext{
${ }^{a}$ Electronic mail: kin@amp.i.kyoto-u.ac.jp

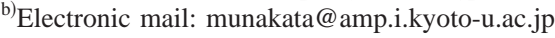

as the first term on the right hand side of Eq. (2), and $F_{\text {ex }}$ is expressed as an expansion around the uniform liquid state $n(\mathbf{r})=n_{0}$. Then $F[n]$ is given by

$$
\begin{aligned}
F[n] \equiv & F_{\mathrm{id}}+F_{\mathrm{ex}}=k_{\mathrm{B}} T \int d \mathbf{r} n(\mathbf{r})\left\{\ln \left[n(\mathbf{r}) \Lambda^{d}\right]-1\right\} \\
& -k_{\mathrm{B}} T \sum \frac{1}{M !} \int d \mathbf{r}_{1} \cdots \int d \mathbf{r}_{M}\left\{C_{M}\left(\mathbf{r}_{1}, \ldots, \mathbf{r}_{M}\right)\right\} \\
& \times\left\{n\left(\mathbf{r}_{1}\right)-n_{0}\right\} \cdots\left\{n\left(\mathbf{r}_{M}\right)-n_{0}\right\},
\end{aligned}
$$

where $\Lambda$ and $C_{M}$ denote the thermal wavelength and the $M$-th-order direct correlation function, respectively.

If $F_{\text {ex }}$ is truncated after $M=2$ as is usually done, we have the free energy functional

$$
\begin{aligned}
F[n] \simeq & k_{\mathrm{B}} T \int d \mathbf{r} n(\mathbf{r})\left\{\ln \left[n(\mathbf{r}) \Lambda^{3}\right]-1\right\} \\
& -\frac{k_{\mathrm{B}} T}{2} \int d \mathbf{r} \int d \mathbf{r}^{\prime}\left[n(\mathbf{r})-n_{0}\right] \\
& \times C_{2}\left(\left|\mathbf{r}-\mathbf{r}^{\prime}\right|\right)\left[n\left(\mathbf{r}^{\prime}\right)-n_{0}\right],
\end{aligned}
$$

which was employed by Ramakrishnan and Yussouff to investigate a liquid-crystal transition. ${ }^{5}$ The $C_{2}(r)$ is related to the two-body total correlation function $h_{2}(r) \equiv g_{2}(r)-1$ by the Ornstein-Zernike (OZ) relation, ${ }^{1}$

$$
h_{2}(r)=C_{2}(r)+n_{0} \int d \mathbf{r}^{\prime} C_{2}\left(\left|\mathbf{r}-\mathbf{r}^{\prime}\right|\right) h_{2}\left(r^{\prime}\right) .
$$

We note that the second-order direct correlation function $C_{2}(r)$ multiplied by $-k_{\mathrm{B}} T$ can be interpreted as the twobody effective interaction.

Inserting the free energy functional form (3) into the variational equation (1), we immediately obtain from $n(\mathbf{r})$ $=n_{0} g_{2}(r)$

$\ln g_{2}(r)=-\phi(r) /\left(k_{\mathrm{B}} T\right)+n_{0} \int d \mathbf{r}^{\prime} C_{2}\left(\left|\mathbf{r}-\mathbf{r}^{\prime}\right|\right) h_{2}\left(r^{\prime}\right)$,

which is equivalent to the HNC equation. ${ }^{1}$ The equation set (4) and (5) can be solved numerically to yield the theoretical expression for $g_{2}(r)$. 
In our previous paper we approximated $F_{\text {ex }}$ by including up to the $M$-th-order terms in Eq. (2) $[M(>2)$-body DFT $]$ and derived the generalized HNC equation, which was to be supplemented with the up to $M$-th-order Ornstein-Zernike relations to obtain a closed set of integral equations. ${ }^{10}$ For the case of $M=3$, we tried to solve the HNC theory and could present rather imperfect numerical results because of various difficulties in our numerical calculations. ${ }^{10}$ In similar efforts to understand and to take into account effects of higher order correlations in liquids, Verlet introduced the PY2 or HNC2 equation by extending the systematic functional expansion method due to Percus. ${ }^{11}$ Similarly there have been several attempts to understand the three-body correlation in a fluid. ${ }^{12-14}$

As is anticipated, it is a demanding task to obtain numerical solutions of integral equations including the threebody correlation function. The purpose of this paper is to try again numerical solutions of the three-body HNC theory and present more reliable numerical solution to enable evaluation of our three-body HNC theory. The organization of this paper is as follows. In Sec. II, we summarize the three-body HNC theory and in Sec. III, after explaining our method of numerical solutions, we present numerical results of twoand three-body correlation functions. Finally in Sec. IV we conclude this paper.

\section{FORMALISM OF THREE-BODY HNC THEORY}

In order to make this paper self-contained, here we briefly summarize $M=3$ HNC (HNC3) theory and write down equations necessary for an explanation of our numerical algorithm. For the case of $M=3$, we have a set of equations to determine the two- and three-body correlation functions $g_{2}(1,2)$ and $g_{3}(1,2,3)$ with 1 denoting the position vector $\mathbf{r}_{1}$. As to $g_{3}(1,2,3)$, we can express it as $g_{3}(1,2,3)$ $=g_{1}(2 \mid 1) g_{1}(3 \mid 1,2)$, where $g_{1}(3 \mid 1,2)$ represents the onebody distribution function at 3 when two particles are located at 1 and 2 , and $g_{1}(2 \mid 1)$ is identical with the radial distribution function $g_{2}(1,2)$. According to our previous paper, the HNC3 theory consists of the following equations:

$$
\begin{aligned}
\ln g_{1}(2 \mid 1) & \\
= & -\phi(1,2) /\left(k_{\mathrm{B}} T\right)+n_{0} \int d 1^{\prime} C_{2}\left(1^{\prime}, 2\right) h_{1}\left(1^{\prime} \mid 1\right) \\
& +\left(n_{0}^{2} / 2\right) \int d 1^{\prime} \int d 2^{\prime} C_{3}\left(1^{\prime}, 2^{\prime}, 2\right) h_{1}\left(1^{\prime} \mid 1\right) h_{1}\left(2^{\prime} \mid 1\right) \\
\equiv & -\phi(1,2) /\left(k_{\mathrm{B}} T\right)+C^{(2)}(2 \mid 1)+B^{(2)}(2 \mid 1)
\end{aligned}
$$

$\ln g_{1}(3 \mid 1,2)$

$$
\begin{aligned}
= & -(\phi(1,3)+\phi(2,3)) /\left(k_{\mathrm{B}} T\right) \\
& +n_{0} \int d 1^{\prime} C_{2}\left(1^{\prime}, 3\right) h_{1}\left(1^{\prime} \mid 1,2\right) \\
& +\left(n_{0}^{2} / 2\right) \int d 1^{\prime} \int d 2^{\prime} C_{3}\left(1^{\prime}, 2^{\prime}, 3\right) \\
& \times h_{1}\left(1^{\prime} \mid 1,2\right) h_{1}\left(2^{\prime} \mid 1,2\right) \\
\equiv & -(\phi(1,3)+\phi(2,3)) /\left(k_{\mathrm{B}} T\right)+C^{(3)}(3 \mid 1,2) \\
& +B^{(3)}(3 \mid 1,2),
\end{aligned}
$$

where $h_{1}(2 \mid 1) \equiv g_{1}(2 \mid 1)-1$ and $h_{1}(3 \mid 1,2) \equiv g_{1}(3 \mid 1,2)-1$. Here $C^{(m)}$ and $B^{(m)}(m=2,3)$ are called an $m$-body convolution and bridge function, respectively. ${ }^{10}$ Neglecting the $B^{(2)}$ in Eq. (6), we immediately obtain the usual (two-body) HNC equation (5). The $B^{(2)}$ represents the potential field at 2 produced by particles at $1^{\prime}$ and $2^{\prime}$ through the $C_{3}\left(1^{\prime}, 2^{\prime}, 2\right)$. Similarly to the $C_{2}$, the third-order direct correlation function $C_{3}$ multiplied $-k_{\mathrm{B}} T$ can be regarded as the effective three-body interaction.

We next turn to the generalized OZ relations between the functions $g_{2}, g_{3}$ and $C_{2}, C_{3}$. It is useful to express the twoand three-body $\mathrm{OZ}$ relations in Fourier space because of their convolution structures:

$$
\begin{aligned}
& \hat{h}_{2}(q)=\hat{C}_{2}(q)\left(1+n_{0} \hat{h}_{2}(q)\right), \\
& \hat{H}\left(\mathbf{q}_{1}, \mathbf{q}_{2}\right)=\hat{C}_{3}\left(\mathbf{q}_{1}, \mathbf{q}_{2}\right) G\left(\mathbf{q}_{1}, \mathbf{q}_{2}\right),
\end{aligned}
$$

where

$$
\begin{aligned}
G\left(\mathbf{q}_{1}, \mathbf{q}_{2}\right) \equiv & \left(1+n_{0} \hat{h}_{2}\left(q_{1}\right)\right)\left(1+n_{0} \hat{h}_{2}\left(q_{2}\right)\right) \\
& \times\left(1+n_{0} \hat{h}_{2}\left(\left|\mathbf{q}_{1}+\mathbf{q}_{2}\right|\right)\right),
\end{aligned}
$$

and $\hat{C}_{3}\left(q_{1}, q_{2}\right)$ represents the Fourier transform of $C_{3}(1,2,3)$ with 3 taken to be the origin of the coordinate system. Similarly, $\hat{H}\left(q_{1}, q_{2}\right)$ represents the Fourier transform of the function $H(1,2,3)$, defined by

$$
\begin{aligned}
H(1,2,3) \equiv & h_{3}(1,2,3)-h_{2}(1,2)-h_{2}(2,3)-h_{2}(1,3) \\
& -\left[h_{2}(1,2) h_{2}(2,3)+h_{2}(1,3) h_{2}(3,2)\right. \\
& \left.+h_{2}(2,1) h_{2}(1,3)\right] \\
& -n_{0} \int d 4 h_{2}(1,4) h_{2}(2,4) h_{2}(3,4),
\end{aligned}
$$

where $h_{3}(1,2,3) \equiv g_{3}(1,2,3)-1$. In summary, we now have the self-consistent set of HNC3 equations, Eqs. (6), (7), (8) and (9) to determine four unknown functions $g_{2}, C_{2}, C_{3}$ and $g_{3}$ or $g_{1}(3 \mid 1,2)$.

\section{NUMERICAL STUDY OF THREE-BODY HNC THEORY}

\section{A. Numerical calculation details}

The solution of the integral equation such as the HNC equation has been usually obtained numerically. To this end, the iterative scheme, which is generally called the Picard method, is introduced. ${ }^{1}$ This method can be applied to our HNC3 theory because of its similar structure to the HNC theory. The procedure used here is the following: (i) take the initial direct correlation functions $C_{m \text {,in }}(m=2,3)$ approximated by the low density limit $n_{0} \rightarrow 0$; (ii) calculate the functions $h_{2}$ and $h_{3}$ through the OZ relations (8) and (9); (iii) obtain the new functions $h_{1}(2 \mid 1)$ and $h_{1}(3 \mid 1,2)$ by calculating the right-hand side of Eqs. (6) and (7); (iv) calculate the new direct correlation functions $C_{m \text {,out }}$ from the $h_{1}$ functions obtained in step (iii) through the OZ relations; (v) take the next input direct correlation functions as 


$$
C_{m, \text { in }}^{\text {new }}=\left(1-\alpha_{m}\right) C_{m, \text { in }}+\alpha_{m} C_{m, \text { out }} \quad(m=2,3),
$$

where $\alpha_{m}$ is the parameter denoting a mixing weight; (vi) iterate (ii)-(v) until convergence is achieved. In order to judge the convergence, we define the norms $N_{m}(m=2,3)$ as

$$
N_{m} \equiv \frac{\left\|C_{m, \text { out }}-C_{m, \text { in }}\right\|}{\left\|C_{m, \text { in }}\right\|},
$$

and monitor their changes at each iteration.

The system considered here is a one-dimensional (1D) liquid with the pairwise interaction $\phi(r)=\epsilon(\sigma / r) .{ }^{12}$ The units of length and temperature are taken as $l \equiv 1 / n_{0}$ and $T^{*} \equiv\left(k_{\mathrm{B}} T / \epsilon\right)(l / \sigma)^{12}$, respectively. Then the thermodynamic state of the system can be characterized by one variable $T^{*}$. We considered two thermodynamic states $T^{*}=10000$ and 50000 , where the radial distribution function is moderately oscillatory. Since we obtained similar results in both cases, we will show the result of only $T^{*}=10000$ in this paper. A numerical calculation was carried out with $\alpha_{2}=0.01$ and $\alpha_{3}=0.1$ in Eq. (12), which was chosen empirically. We fixed the variable 1 at the origin of the coordinate from the invariance of Eqs. (6) and (7) under space translation. For convenience, the variables 2 and 3 are expressed by $x$ and $y$ so that the functions, for example, $g_{2}(1,2)$ and $C_{3}(1,2,3)$ will be expressed simply by $g_{2}(x)$ and $C_{3}(x, y)$, respectively. The variables $x$ and $y$ are considered in the range $|x|,|y| \leqslant 10$ with 512 mesh-points, convenient for use of the fast Fourier transform (FFT) method.

In our previous approach, we could not achieve convergence in the calculation of $C_{3}$, whose norm $N_{3}$ was observed to increase after about 100 iterations. ${ }^{10}$ The problem was that the $C_{3}$ could not keep its smooth structure in the course of iteration due to the accumulation of numerical errors. The errors turn out to come mainly from the use of the two-dimension discrete Fourier transform. To eliminate this difficulty related to FFT, here we introduce the filter function $f(x, y) \equiv \exp \left(-x^{2} / a^{2}\right) \exp \left(-y^{2} / a^{2}\right)$ with a constant $a$. We multiply the $C_{3}(x, y)$ by the $f(x, y)$ with $a=6$ at each iteration in order to make $C_{3}(x, y)$ a smooth function. Since the $C_{3}(x, y)$ is a localized function of $x$ and $y$ around the origin $(0,0)$ in the range $|x|,|y| \leq 1$, this procedure does not influence the shape of the $C_{3}(x, y)$.

In Fig. 1, we show the norm changes $N_{m}$ for $T^{*}$ $=10000$ as an iteration step increases. It is seen in Fig. 1 that both $N_{2}$ and $N_{3}$ become the order of $10^{-6}$ after about 15000 iterations and the convergence is fulfilled. In Fig. 2 we compare $C_{2}(x)$ in the low density limit with the one from the HNC3 theory. In Fig. 3, we also plotted $C_{3}(x, y)$ in the low density limit (a) and from the HNC3 theory (b). For $C_{2}$ and $C_{3}$, the initial direct correlation functions are seen to considerably "grow up" in a similar way due to the pairwise interaction, leading to rather strong effective repulsion for the small separation of particles.

\section{B. Results}

We performed molecular dynamics (MD) simulations of a 1D liquid with 4000 particles and obtained $g_{2}(x)$ at $T^{*}$ $=10000^{2}$. In Fig. 4, we show $g_{2}(x)$ at $T^{*}=10000$. It is well known and also shown here that the $g_{2}(x)$ from the

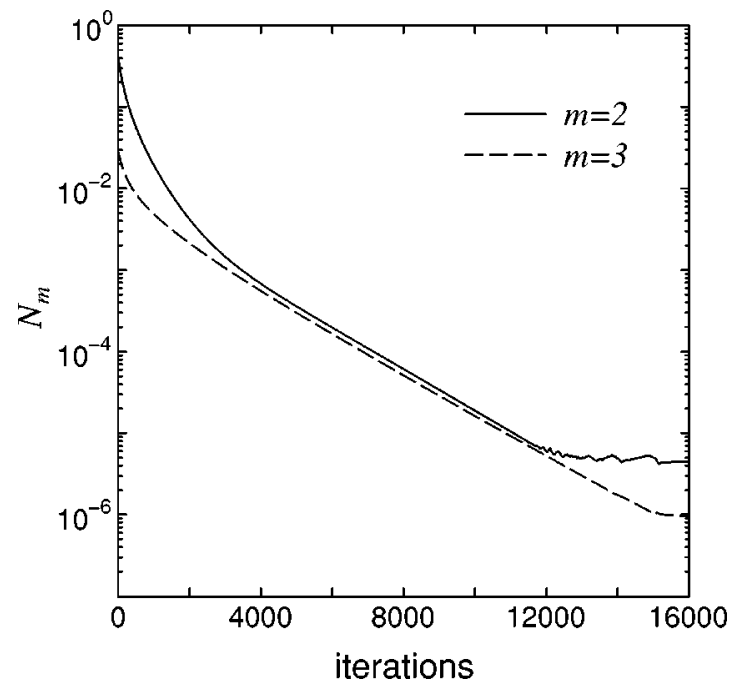

FIG. 1. The norm variation as a function of iteration steps for $C_{2}$ (solid curve) and $C_{3}$ (dashed curve).

HNC equation has a higher first peak and a more compressed structure than the experimental one. On the other hand, it is noted that the HNC3 theory gives better $g_{2}(x)$ with respect to the height of the first peak and the phase of the oscillation.

This improvement of the $g_{2}(x)$ is attributed to the threebody effect, which is explicitly taken into account through the $B^{(2)}(x)$ in Eq. (6). In order to quantify the three-body effect, let us turn to the bridge function $B^{(2)}(x)$ and the convolution function $C^{(2)}(x)$ in Eq. (6). In Fig. 5, we plot the combination $C^{(2)}(x)+B^{(2)}(x)$ in Eq. (6) and $C^{(2)}(x)$ from the HNC theory and the potential term $\phi(x) / T^{*}$ for the purpose of comparison. From Fig. 5, we notice that the $C^{(2)}(x)+B^{(2)}(x)$ from the HNC3 theory is smaller than the $C^{(2)}(x)$ from the HNC theory at $x \simeq 0.5$ where a particle contacts with a core. This change induces the improvement

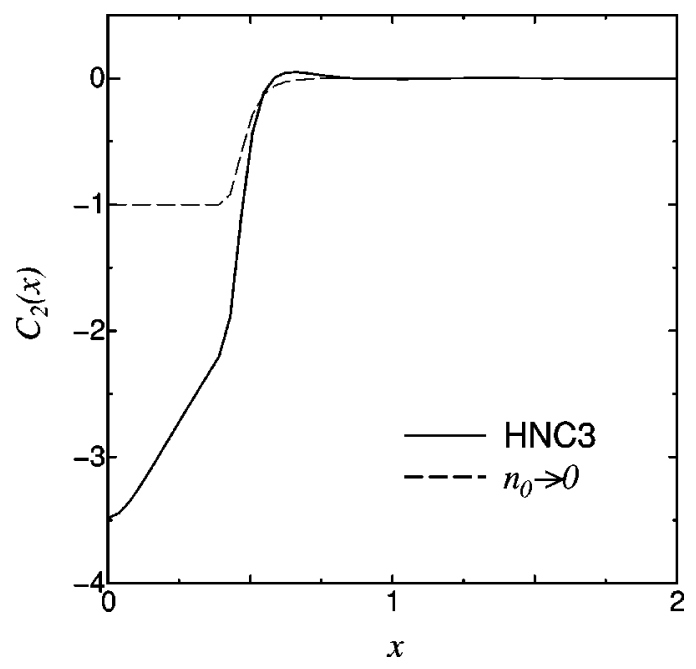

FIG. 2. The second-order direct correlation function $C_{2}(x)$ at $T^{*}=10000$ from the HNC3 theory (solid curve) and in the low density limit (dashed curve). 


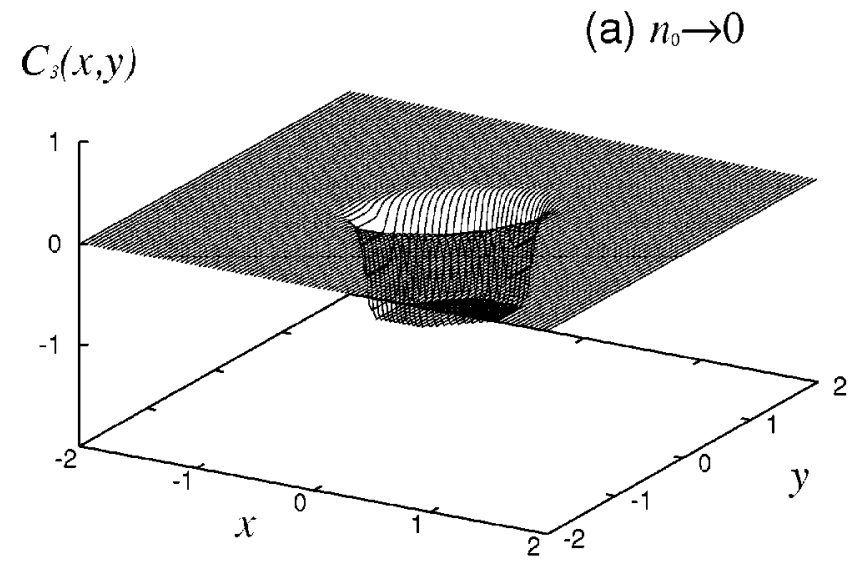

(b) $\mathrm{HNC3}$

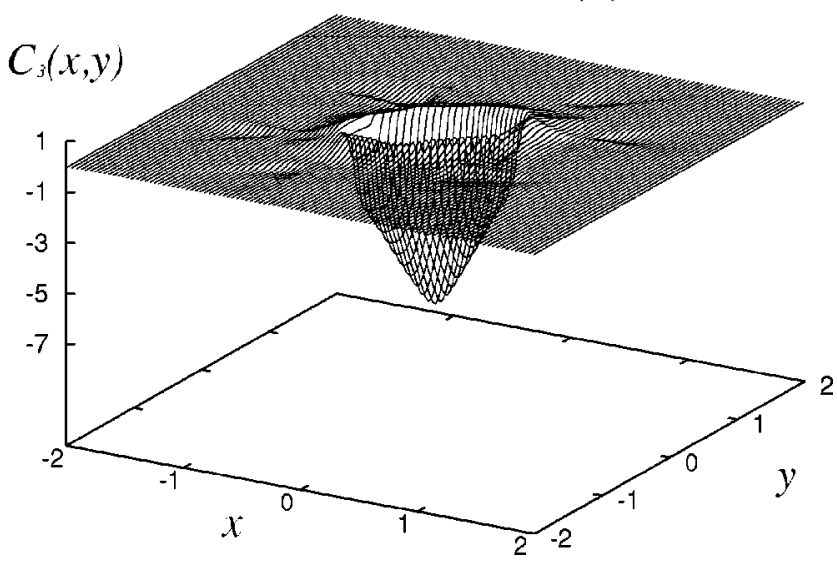

FIG. 3. The third-order direct correlation function $C_{3}(x, y)$ at $T^{*}=10000$ in the low density limit (a) and from the HNC3 theory (b).

of the $g_{2}(x)$ with respect to the height of the first peak. In addition to this, Fig. 5 shows that there is a moderate improvement as to the oscillation phase between the HNC3 and the $\mathrm{HNC}$ theories.

Finally we comment on the Kirkwood superposition approximation (SA). ${ }^{15}$ Kirkwood approximated the three-body

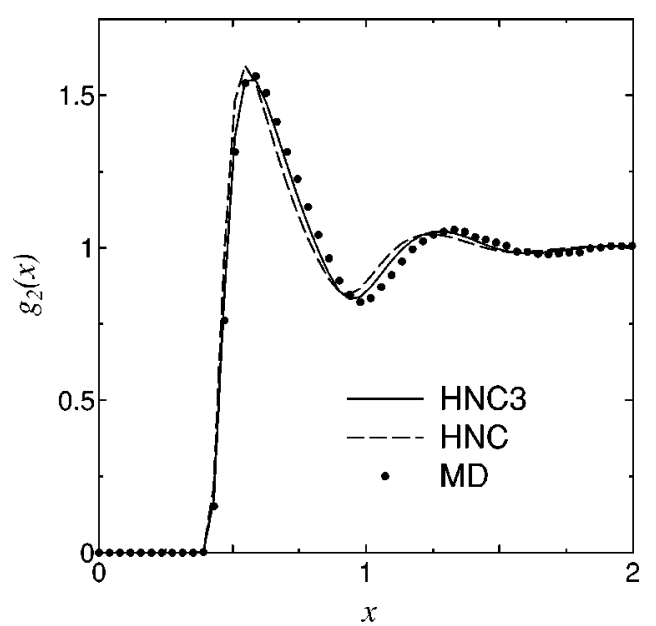

FIG. 4. The radial distribution function $g_{2}(x)$ at $T^{*}=10000$ from the HNC3 theory (solid curve), the HNC theory (dashed curve) and MD simulation (closed circles).

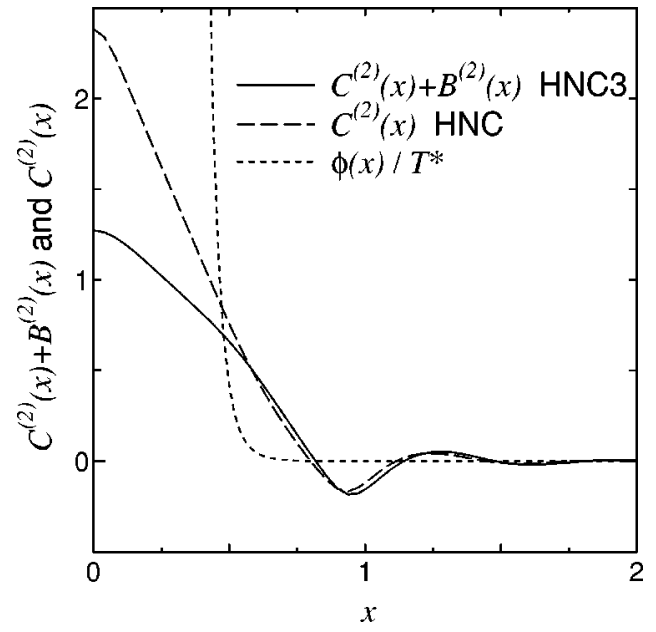

FIG. 5. $C^{(2)}(x)+B^{(2)}(x)$ (solid curve) from the HNC3 theory, $C^{(2)}(x)$ (dashed curve) for the HNC theory, and the potential term $\phi(x) / T^{*}$ (dotted curve).

correlation function as the product of the two-body correlation functions as

$$
g_{3}(1,2,3) \simeq g_{2}(1,2) g_{2}(2,3) g_{2}(3,1) .
$$

One obtains the Born-Green equation for $g_{2}(x)$ by applying this approximation to the Yvon-Born-Green hierarchy. ${ }^{1}$ Once this factorized approximation is employed, we can obtain the third-order direct correlation function by inserting the $\mathrm{SA}_{3}$ to the three-body OZ relation (9). We thereby have another self-consistent closure ( $\mathrm{HNC} 3+\mathrm{SA})$ consisting of Eqs. (6), (8), (9) and (14) without recourse to Eq. (7). It should be noted that the bridge function $B^{(2)}$ in Eq. (6) is calculated by the $C_{3}$ approximated with the SA. We carried out the numerical calculation of the $\mathrm{HNC} 3+\mathrm{SA}$ with the iterative method explained before. In Fig. 6, we show the $g_{2}(x)$ at $T^{*}=10000$ for the HNC3 $+\mathrm{SA}, \mathrm{HNC}$, and MD, which indicate that the $\mathrm{HNC} 3+\mathrm{SA}$ theory improves the HNC theory similarly but slightly to lesser extent than the HNC3 theory does.

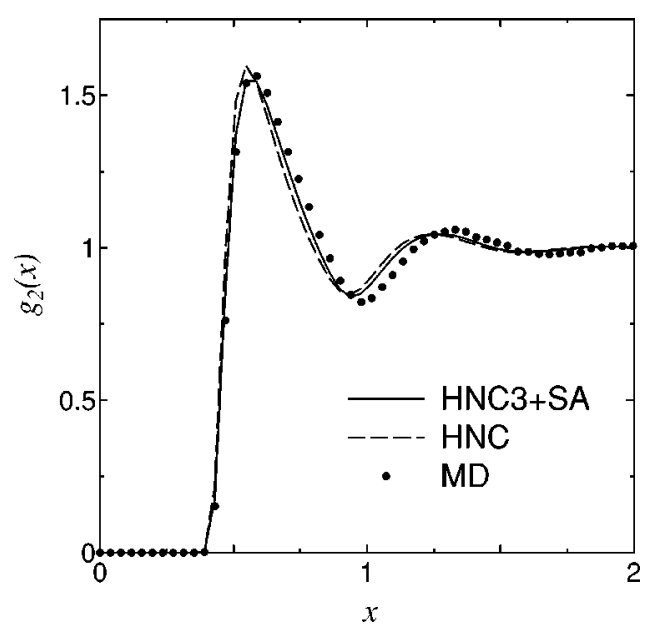

FIG. 6. The radial distribution function $g_{2}(x)$ at $T^{*}=10000$ from the HNC3 + SA theory (solid curve), the HNC theory (dashed curve) and MD simulation (closed circles). 


\section{CONCLUSIONS}

In this paper, we discussed the HNC3 theory derived before based on the three-body DFT and Percus' idea. We solved a rather complicated set of equations, which consists of Eqs. (6), (7), (8) and (9), to determine the two- and threebody correlation functions self-consistently. For the 1D softrod system the HNC3 theory was shown to improve the HNC theory with respect to the height of the first peak and the phase relations of $g_{2}(x)$. Effects on the radial distribution function of the third-order direct correlation function $C_{3}$ were discussed through the convolution and bridge functions.

It should be noted that the system considered in the present work is at rather high (low) temperature (density). Furthermore, we studied only a 1D system in our numerical calculations. Numerical studies of the HNC theory for a 3D system seems to be a real challenge, which is worthwhile because three-body correlations play important roles in a dense fluid like a supercooled liquid or a glass. We hope that the $M$-body DFT will also lead to new insights into classical many-body problems.
${ }^{1}$ J. P. Hansen and I. R. McDonald, Theory of Simple Liquids (Academic, New York, 1986).

${ }^{2}$ M. P. Allen and D. J. Tildesley, Computer Simulations of Liquids (Clarendon, Oxford, 1987).

${ }^{3}$ J. M. J. van Leeuwen, J. Groeneveld, and J. de Boer, Physica (Amsterdam) 25, 792 (1959); T. Morita and H. Hiroike, Prog. Theor. Phys. 23, 1003 (1960).

${ }^{4}$ For reviews, see A. D. J. Haymet, Annu. Rev. Phys. Chem. 38, 89 (1987); D. W. Oxtoby, in Liquid, Freezing, and the Glass Transition, edited by J. P. Hansen, D. Levesque, and J. Zimm-Justin (Elsevier, New York, 1990).

${ }^{5}$ T. V. Ramakrishnan and M. Yussouff, Phys. Rev. B 19, 2775 (1979).

${ }^{6}$ A. D. Haymet and D. W. Oxtoby, J. Chem. Phys. 74, 2559 (1981); D. W. Oxtoby and A. D. Haymet, ibid. 76, 6262 (1982).

${ }^{7}$ Y. Singh, J. P. Stoessel, and P. G. Wolynes, Phys. Rev. Lett. 54, 1059 (1985).

${ }^{8}$ D. Chandler, J. D. Mccoy, and S. J. Singer, J. Chem. Phys. 85, 5971 (1986); T. Munakata, S. Yoshida, and F. Hirata, Phys. Rev. E 54, 3687 (1996).

${ }^{9}$ J. Percus, in The Equilibrium Theory of Classical Fluids, edited by H. L. Frisch and J. L. Lebowitz (Benjamin, New York, 1964).

${ }^{10}$ T. Munakata and K. Kim, J. Chem. Phys. 113, 3975 (2000).

${ }^{11}$ L. Verlet, Physica (Amsterdam) 30, 95 (1964); 31, 959 (1965).

${ }^{12}$ J. L. Barrat, J. P. Hansen, and G. Pastore, Phys. Rev. Lett. 58, 2075 (1987).

${ }^{13}$ P. Attard, J. Chem. Phys. 93, 7301 (1990).

${ }^{14}$ E. Lomba, S. Jorge, and M. Álvarez, Phys. Rev. E 63, 011203 (2000),

${ }^{15}$ J. G. Kirkwood, J. Chem. Phys. 3, 300 (1935). 